\title{
Exciton trapping in one-dimensional systems with correlated disorder
}

\author{
Francisco Domínguez-Adame and Bianchi Méndez \\ Departamento de Física de Materiales, Facultad de Físicas, Universidad Complutense, E-28040 Madrid, Spain \\ Angel Sánchez \\ Escuela Politécnica Superior, Universidad Carlos III de Madrid, E-28913 Leganés, Madrid, Spain \\ Enrique Maciá* \\ Departamento de Física de Materiales, Facultad de Fúsicas, Universidad Complutense, E-28040 Madrid, Spain
}

(Received 28 July 1993; revised manuscript received 1 September 1993)

\begin{abstract}
Numerical investigations of the trapping of Frenkel excitons in one-dimensional lattices with interstitial traps randomly placed in pairs are presented. The probabilities of finding the exciton both in the $q=0$ mode $P(t)$ and in any mode $Q(t)$ have been obtained following the numerical approach recently developed by Huber and Ching [Phys. Rev. B 42, 7718 (1990)] for unpaired traps. We have found that the pairing of traps enhances both probabilities at all times, in comparison with lattices containing the same fraction of unpaired traps. We suggest that this behavior is related to the occurrence of larger segments of the lattice that are free of traps, where there exists a major contribution of the slowly decaying modes.
\end{abstract}

\section{INTRODUCTION}

A great deal of theoretical work has been devoted to the study of excitations in disordered solids. However, the understanding of quasiparticle dynamics in disordered systems is far from complete. The investigations generally split into two different topics: transport properties and spectroscopy. Concerning the first topic, some new phenomena have been found in relation to quasiparticle localization. A number of recent works strongly suggest that the occurrence of correlated disorder introduces a competition between the long-range disorder and the short-range correlation causing long-range transport. Thus, for instance, electron delocalization appears in tight-binding Hamiltonians in which the on-site energy takes on one of two possible values, one of which is assigned at random to pairs of lattice sites (the so-called random dimer model ${ }^{1-5}$ ). Moreover, delocalization by correlation is not restricted to electronic random systems; for instance, the occurrence of delocalized vibrations by paired disorder has recently been demonstrated in classical random chains by means of multifractal analysis. ${ }^{6}$ In contrast to this progress, much less attention has been paid to the spectroscopy of correlated disordered systems.

Trapping of Frenkel excitons in one-dimensional systems with randomly distributed (uncorrelated) traps was earlier studied by Hemenger et al. ${ }^{7,8}$ obtaining exact results for a number of special cases. More recently, Huber and Ching have considered both interstitial ${ }^{9}$ and substitutional ${ }^{10}$ traps (an explanation of the basic differences between the effects of these two kind of traps may be found in Ref. 11), where the effects of trapping are introduced through a non-Hermitian decay term in the Hamiltonian. In this paper we investigate the dynamics of one-dimensional lattices whose excited states are
Frenkel excitons, in which the coherent quantum transport is affected by the presence of pairs (correlated) of interstitial traps placed at random in an otherwise perfect lattice. We believe that the study of this model is beyond the formal study of the exciton dynamics. Indeed, our model could provide insight into the optical properties of some polymers with active molecules (for instance, it is known that polyaniline can be mapped onto a random dimer model $^{3}$ ); besides, it could be extended to three dimensions in order to explain some basic features of luminescence in alkali halide mixed crystals (assuming the validity of the Frenkel-exciton approach in these solids), as in the case of a dimer emission band originating from the localized exciton created at a pair of $\mathrm{Br}^{-}$ions, as occurs in $\mathrm{KCl}: \mathrm{Br} .^{12}$

\section{MODEL}

The Hamiltonian for the Frenkel-exciton problem in the ordered lattice without traps can be written in tightbinding form with nearest-neighbor interactions as follows (we use units such that $\hbar=1$ ):

$$
\mathcal{H}=V \sum_{k} a_{k}^{\dagger} a_{k}+T \sum_{k}\left(a_{k}^{\dagger} a_{k+1}+a_{k+1}^{\dagger} a_{k}\right) .
$$

Here $a_{k}$ and $a_{k}^{\dagger}$ are the exciton annhilitation and creation operators in the site representation, respectively. $V$ is the single-ion transition frequency, and $T$ is the nearestneighbor coupling (the exciton bandwidth is $2|T|$ ).

As mentioned above, we shall be concerned with the dynamics of Frenkel excitons when interstitial traps are introduced in the ordered lattice. Interstitial traps do not replace specific sites: Trapping will occur when particles 
at sites close to the trap fall into it by emitting a phonon. We assume that either temperature is low and trap is deep enough to avoid detrapping processes. The presence of traps is taken into account by adding a non-Hermitian decay term of the form ${ }^{9}$

$$
-i \sum_{k} \Gamma_{k} a_{k}^{\dagger} a_{k}
$$

to the Hamiltonian (1). Sum runs over lattice sites and $\Gamma_{k}$ is nonzero only if the $k$ th site has a trap associated with it. This parameter gives the probability of finding the $k$ th ion in the excited state as $\exp \left(-2 \Gamma_{k} t\right)$ whenever the coupling between ions is negligible (radiative transitions will be ignored throughout the paper).

The correlated disorder is introduced as follows: We suppose that all traps are identical so that $\Gamma_{k} \equiv \Gamma$, and they are randomly distributed along the lattice but only appear in pairs of neigboring sites (the correlation length equals the lattice spacing). Thus, we define the fraction of traps $c$ as the ratio between the number of sites with a trap associated with it and the total number of sites in the lattice. Following Refs. 9 and 13, trapping of a $q=0$ exciton created by a pulsed optical excitation in a lattice with $N$ sites is described by a set of correlation functions

$$
G_{k}(t)=\frac{1}{\sqrt{N}} \sum_{j}\left\langle 0\left|a_{k}(t) a_{j}^{\dagger}\right| 0\right\rangle,
$$

where $|0\rangle$ denotes the exciton vacuum state and $a_{k}(t)=$ $\exp (i \mathcal{H} t) a_{k} \exp (-i \mathcal{H} t)$. The function $G_{k}(t)$ obeys the equation of motion

$$
i \frac{d}{d t} G_{k}(t)=\sum_{j} H_{k j} G_{j}(t),
$$

with the initial condition $G_{k}(0)=1 / \sqrt{N}$. The diagonal elements of the tridiagonal matrix $H_{j k}$ are $V-i \Gamma_{k}$, whereas off-diagonal elements are simply given by $T$. The equation of motion is a discrete Schrödinger-like equation on a lattice, and standard numerical techniques may be applied to obtain the solution. Once the equation of motion is solved, the following two functions are calculated:

$$
P(t)=\frac{1}{N}\left|\sum_{k} G_{k}(t)\right|^{2}
$$

and

$$
Q(t)=\sum_{k}\left|G_{k}(t)\right|^{2} .
$$

These two functions characterize the exciton dynamics. ${ }^{9}$ $P(t)$ is identified with the probability of finding an exciton in the $q=0$ mode at time $t$, whereas $Q(t)$ is the probability of finding the exciton in any mode at time $t$ (survival probability). Hence, it is clear that $P(t) \leq Q(t)$ at all times.

\section{NUMERICAL RESULTS AND DISCUSSIONS}

We have numerically solved the equation of motion (4) for chains of $N=1000$ sites using an implicit integration scheme. Energy will be measured in units of $T$, whereas time will be expressed in units of $T^{-1}$. The maximum integration time and the integration time step were 25 and $5 \times 10^{-3}$, respectively; smaller time steps led to the same results. Since we are mainly interested in the effects of the configuration of the random system (correlated disorder) rather than in the effects of the different parameters on the trapping process, we have set $V=-T=\Gamma=1$ hereafter. The fraction of traps ranged from 0.01 up to 1.0 , and for each lattice a random distribution of paired traps was chosen. In addition, lattices with uncorrelated (unpaired) traps have been studied and compared with lattices containing the same fraction of correlated traps. This enables us to separate the effects merely due to trapping in one dimension from those that manifest the peculiarities of the correlation between random traps.

In our computations we found that $P(t)$ decays faster as the fraction of traps increases, as shown in Fig. 1. This behavior is also obtained in the case of uncorrelated traps. ${ }^{9}$ This is expected, since trapping depletes the $q=0$ mode in both cases. Although not shown in the figure, we have checked that $P(t) \sim \exp (-2 \Gamma t)$ in the limit $c \rightarrow 1$. In this limit the trap distribution exhibit translational symmetry so there is no scattering by disorder. Thus $q=0$ is the unique present mode and the trapping is simply exponential. ${ }^{13}$ For any other fraction of traps randomness occurs and disorder causes a nonexponential decay, as seen in Fig. 1. Similar comments apply to the survival probability $Q(t)$. The corresponding results are presented in Fig. 2. It is observed that this probability also decays nonexponentially (except for the case $c=1$, which is not shown in the figure). The main difference is the decay rate of $P(t)$ and $Q(t)$ for the same fraction of traps (note the different vertical scale in Figs. 1 and 2) showing that $P(t) \leq Q(t)$ at all times.

The above results have been obtained for a single realization. An important and nontrivial question is whether these results strongly depend on each particular realization of the system or not. If different realizations of the system yield rather different values, then it would make no sense to deduce any general behavior of $P(t)$ and

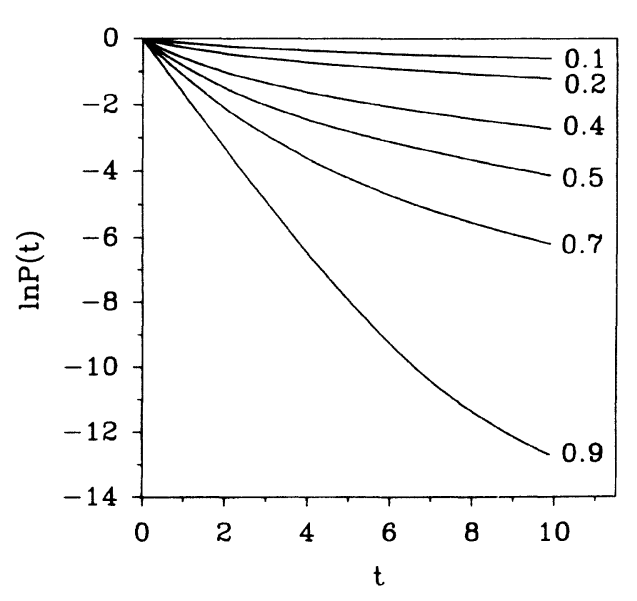

FIG. 1. $\ln P(t)$ versus $t$ for lattices with 1000 sites. The fraction $c$ of paired traps is indicated on each curve. 


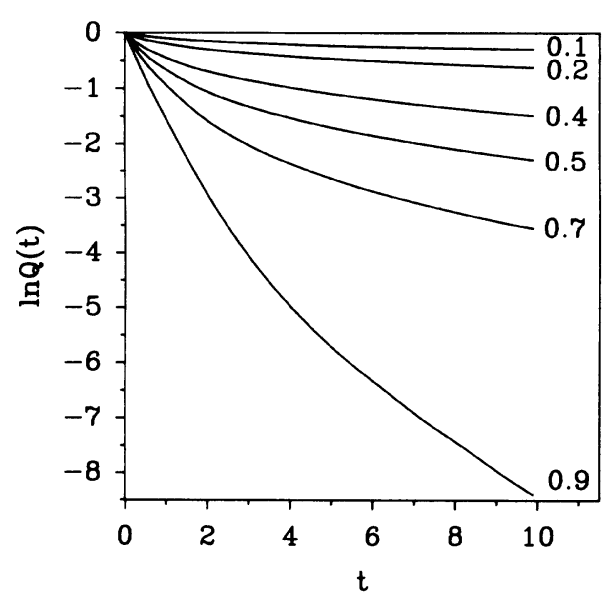

FIG. 2. $\ln Q(t)$ versus $t$ for lattices with 1000 sites. The fraction $c$ of paired traps is indicated on each curve.

$Q(t)$ from a particular realization. It is worth mentioning that other works on exciton trapping in one-dimensional random systems deal with data for a single realization only ${ }^{9,10}$ Therefore, we have investigated this question, generating a number (up to a maximum of 40) of random configurations with a fixed fraction of paired traps. The magnitudes used for averaging were $\ln P(t)$ and $\ln Q(t)$. The reason to average the logarithm of the observable quantities is twofold: First, the numerical values of $P(t)$ and $Q(t)$ become very small for long times, and consequently they can be severely affected by numerical roundoff errors. The second reason is the well-known fact that logarithms usually exhibit better statistical properties than the magnitudes themselves. The relative dispersion of values around the mean was determined at each integration time $t$ as follows:

$$
\zeta(t) \equiv \sqrt{\frac{\left\langle f^{2}(t)\right\rangle-\langle f(t)\rangle^{2}}{\langle f(t)\rangle^{2}}},
$$

where $\langle\cdots\rangle$ denotes an average over all configurations of $N c$ paired traps and $f(t)$ stands for $\ln P(t)$ or $\ln Q(t)$ correspondingly. Notice that the relative dispersion $\zeta(t)$ is the usual dispersion divided by the mean value of the magnitude. In Fig. 3 we present the results for $c=0.2$ and 40 averages for both $\ln P(t)$ and $\ln Q(t)$. Figures $3(\mathrm{a})$ and 3(b) show, respectively, the plots of $\ln P(t)$ and $\ln Q(t)$ along with the uncertainty band given by their dispersion, and Fig. 3(c) shows the corresponding relative dispersion $\zeta(t)$. It is important to realize that the uncertainty band is indeed small: Its width is much less than the distance to the same curves for slightly different fraction of traps (cf. Figs. 1 and 2 taking into account the different vertical scales). We observe that the relative dispersion increases slightly at short times but remains almost constant at large time. The constant values are about $11 \%$ in both cases, so we are led to the conclusion that different configurations of traps yield very similar results without strong fluctuations.

Having confirmed the main features of the exciton decay due to the presence of paired traps, we compare them with those obtained in one-dimensional lattices with unpaired traps. As mentioned above, this comparison will be carried out for systems with the same fraction of traps. In all cases considered we have observed that the exciton decay is slower in the presence of correlated traps. This is illustrated in Fig. 4 for a fraction of traps $c=0.4$. Hence, our computations show that correlation of traps reduces the depletion of the $q=0$ mode as well as the trapping rate as compared to uncorrelated traps. This is one of the main results of this work. The physical explanation of this behavior is based in the particular spatial distribution displayed by the traps when pairing is present. Let us consider the lattice to be divided in finite segments without traps, while we assume that traps are associated to the ions closest to the ends of the segments. It becomes clear that the average length of the segments is larger when correlation is present, since traps
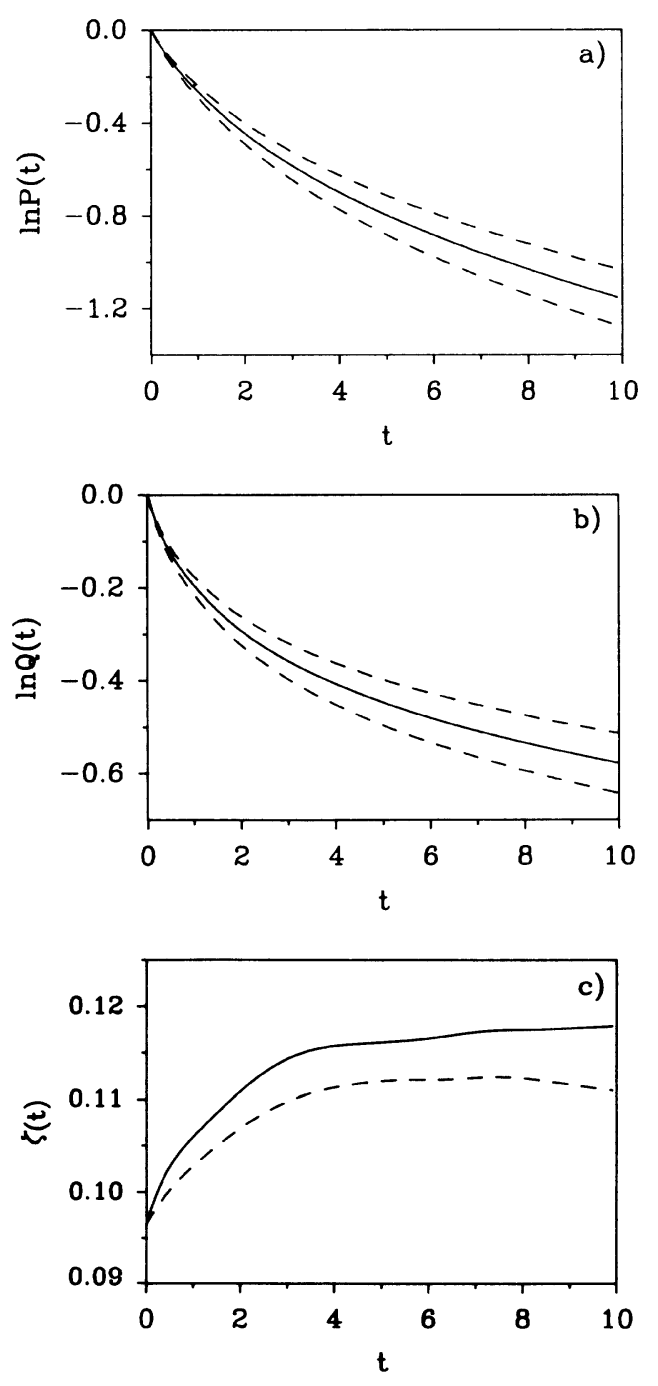

FIG. 3. (a) $\ln P(t)$ and (b) $\ln Q(t)$ (solid lines) along with the uncertainty band given by their dispersions (dashed lines), and (c) relative dispersion of values $\zeta(t)$, as defined in (7), of $\ln P(t)$ (dashed line) and $\ln Q(t)$ (solid line) versus $t$. In all three plots, averages were taken over 40 realizations of systems with a fraction $c=0.2$ of paired traps. 


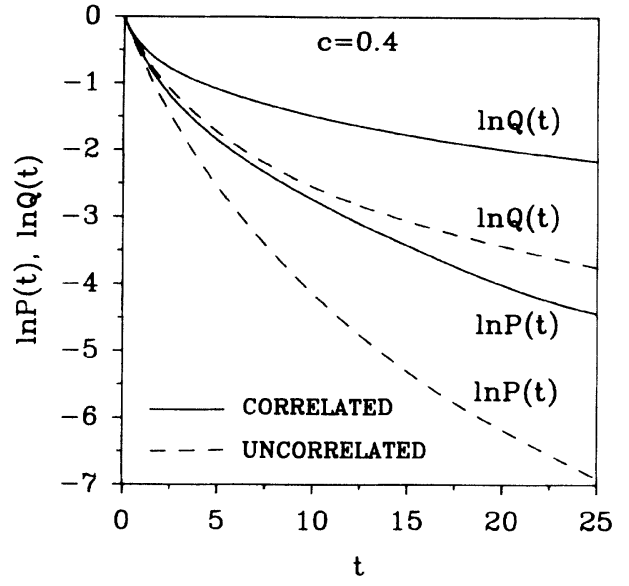

FIG. 4. $\ln P(t)$ and $\ln Q(t)$ versus $t$ for lattices with a fraction $c=0.4$ of correlated (solid lines) and uncorrelated (dashed lines) traps.

always appear in pairs. Hence, the slow decay associated with larger segments without traps causes a reduction of the decay rate when correlation occurs, as observed in our results. To check the validity of this suggestion we have explicitly calculated each term of the sum (6) for lattices with a low fraction $c=0.04$ of paired traps at a fixed time $t=5$, as shown in Fig. 5(a). Results have been compared with those corresponding to lattices with unpaired traps, shown in Fig. 5(b). In both cases we observe deep minima at sites with traps and two small humps close to each minimum. The distance between these two humps gives an estimation of the length of the chain locally affected by the presence of the traps: This length increases slightly with time in the considered range $(0<t<5)$. Remarkably, the separation between the two humps remains almost unchanged, even if two traps are placed together [see insets of Figs. 5(a) and 5(b)]. It is clear that the main contribution to (6) arises from sites without traps, shown as flat regions in Fig. 5, and these flat regions are larger on average in lattices with paired traps, as we have suggested. As further evidence in this direction, we present in Fig. $6 \ln P(t)$ and $\ln Q(t)$ versus $t$ for lattices with a fraction $c=0.1$ of correlated and $c=0.05$ of uncorrelated traps, where the uncorrelated lattice was generated from the correlated one by removing the second trap of each pair (i.e., the spatial distribution of traps or pairs is random, but equal for both cases). As can be seen from the plot, the results are very close for both systems, indicating that the main factor governing the behavior of the system is the presence of segments free of traps.

Finally, we consider the asymptotic long-time decay law of excitons in the presence of traps. This is an interesting problem, and several theoretical ${ }^{11}$ as well as experimental ${ }^{14}$ works have been devoted to find the relaxation law displayed by excitations in one-dimensional systems. In the diffusive limit the survival probability in a $d$-dimensional medium decay as $\sim \exp \left(-A t^{d /(d+2)}\right)$, whereas coherent transport in the presence of traps shows a decay of the form $\sim \exp \left(-A t^{d /(d+3)}\right)$ at long times. ${ }^{15,16}$
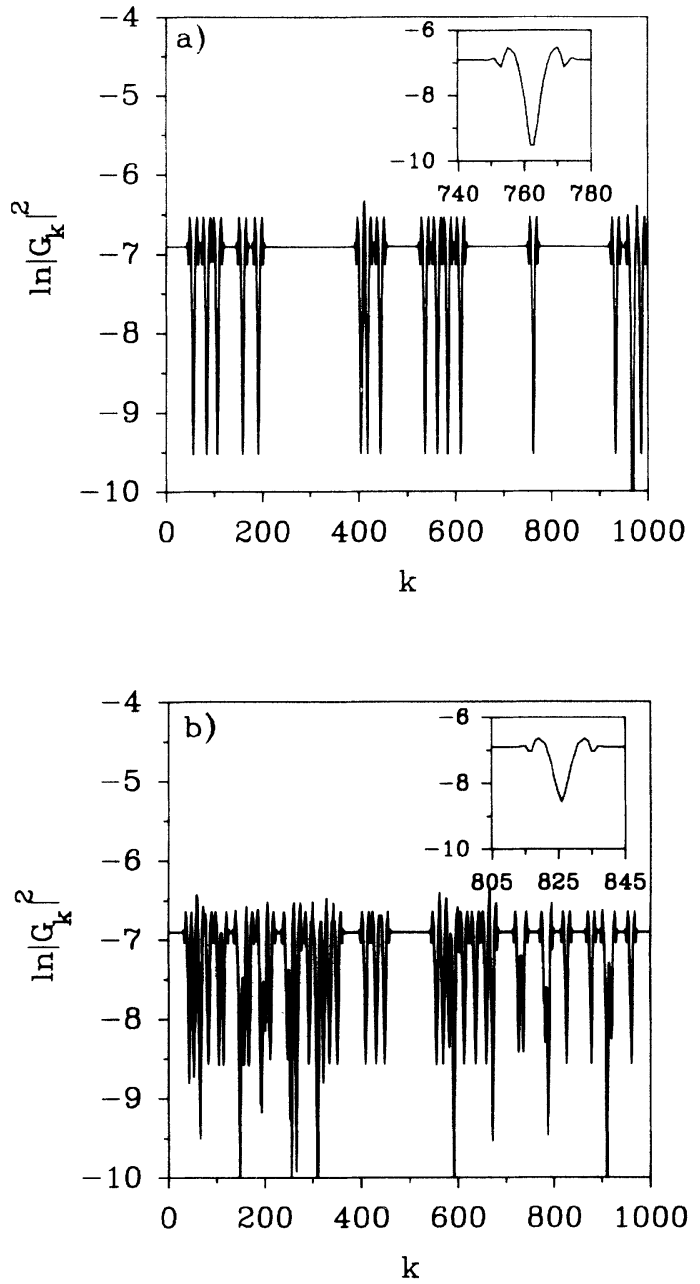

FIG. 5. $\ln \left|G_{k}\right|^{2}$ versus lattice site $k$ at time $t=5$ for lattices with a fraction $c=0.04$ of (a) paired and (b) unpaired traps. The insets show the magnified minima due to traps associated to sites (a) $k=762$ and 763 and (b) $k=826$.

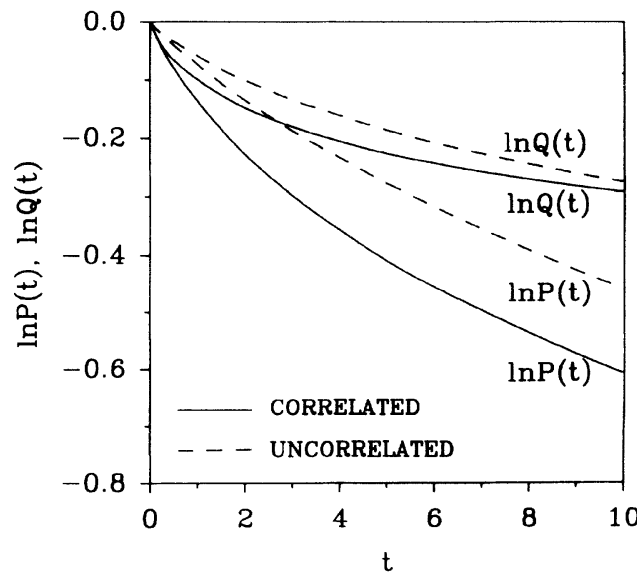

FIG. 6. $\ln P(t)$ and $\ln Q(t)$ versus $t$ for lattices with a fraction $c=0.1$ of correlated (solid lines) and $c=0.05$ of uncorrelated (dashed lines) traps. See text for explanation. 


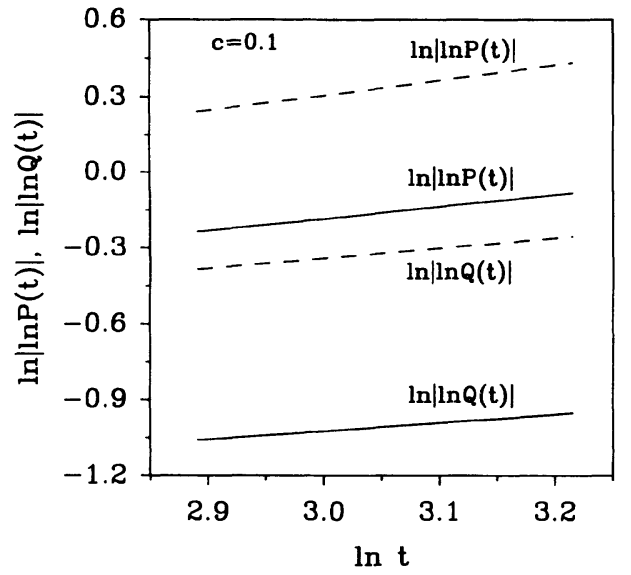

FIG. 7. $\ln |\ln P(t)|$ and $\ln |\ln Q(t)|$ versus $\ln t$ for lattices with a fraction $c=0.1$ of correlated (solid line) and uncorrelated (dashed line) traps.

It is worth mentioning that some authors suggested that this asymptotic behavior is achieved in experimentally accesible times only in one dimension (see Ref. 11 and references therein). We have studied the exciton decay at long times (up to 25) for lattices with uncorrelated, as well as correlated, traps. It must be noticed that our calculations are not in the asymptotic regime, so that a direct comparison with analytical results for $t \rightarrow \infty$ could be inconsistent. We have observed that decay probabilities fit stretched exponentials of the form $P(t) \sim \exp \left(-A t^{\alpha}\right)$ and $Q(t) \sim \exp \left(-B t^{\beta}\right)$ in the range $t=18$ up to 25 . To obtain the parameters $\alpha$ and $\beta$ we have plotted $\ln |\ln P(t)|$ and $\ln |\ln Q(t)|$ versus $\ln t$, as shown in Fig. 7. For a small concentration of traps (up to $c=0.2$ ) we obtained $\alpha \simeq 0.5$ and $\beta \simeq 0.3$ for correlated traps, whereas in the case of uncorrelated traps we obtained $\alpha \simeq 0.6$ and $\beta \simeq 0.4$ over the indicated interval.
These values confirm that disorder correlation reduces the exciton decay rate even at long times.

\section{CONCLUSIONS}

As mentioned in the Introduction, the present study is devoted to obtaining a more complete and general comprehension of the quasiparticle dynamics in onedimensional systems with correlated disorder, which is being investigated extensively at present. In particular, we have numerically investigated the dynamics of Frenkel excitons in one-dimensional ordered lattices with a certain number of paired interstitial traps. The numerical procedure allows one to develop a time analysis of the exciton decay in a relatively simple way. Based on these computations, we have concluded that excitons decay slower when correlation is introduced. The pairing of traps causes the occurrence of larger segments of the lattice, which are free of traps, and the slowly decaying modes associated with them cause the slowdown of the trapping rate. In view of these results, we point out that obtaining analytical results concerning trapping in one dimension with correlated disorder would be an interesting theoretical task. As a final remark from the viewpoint of applications, our findings suggest that studying the optical response to pulsed excitations may be a means to discern the local spatial structure of active centers in solids. Regarding this, we envisage that more complicated groupings of traps may lead to even slower exciton decays. It would then be experimentally relevant to pursue this possibility further.

\section{ACKNOWLEDGMENTS}

A.S. thanks partial financial support from CICyT through project PB92-0248.
* Also at Instituto de Estudios Interdisciplinares, El Guijo, Z4 Galapagar, E-28260 Madrid, Spain

${ }^{1}$ D. H. Dunlap, H.-L. Wu, and P. Phillips, Phys. Rev. Lett. 65, 88 (1990).

${ }^{2}$ H.-L. Wu and P. Phillips, J. Chem. Phys. 93, 7369 (1990).

${ }^{3}$ H.-L. Wu and P. Phillips, Phys. Rev. Lett. 66, 1366 (1991).

${ }^{4}$ P. Phillips and H.-L. Wu, Science 252, 1805 (1991).

${ }^{5}$ A. Sánchez, E. Maciá, and F. Domínguez-Adame, Phys. Rev. B 49, 147 (1994).

${ }^{6}$ F. Domínguez-Adame, E. Maciá, and A. Sánchez, Phys. Rev. B 48, 6054 (1993).

${ }^{7}$ R. P. Hemenger and R. M. Pearlstein, Chem. Phys. 2, 424 (1973).

${ }^{8}$ R. P. Hemenger, K. Lakatos-Lindenberg, and R. M. Pearl- stein, J. Chem. Phys. 60, 3271 (1974).

${ }^{9}$ D. L. Huber and W. Y. Ching, Chem. Phys. 146, 409 (1990).

${ }^{10}$ D. L. Huber, Phys. Rev. B 45, 8947 (1992).

${ }^{11}$ P. E. Parris, Phy. Rev. B 40, 4928 (1989).

${ }^{12}$ K. Tanaka, K. Kan'no, and Y. Nakai, J. Phys. Soc. Jpn. 59, 1474 (1990).

${ }^{13}$ D. L. Huber and W. Y. Ching, Phys. Rev. B 42, 7718 (1990).

${ }^{14}$ I. G. Hunt, D. Bloor, and B. Movaghar, J. Phys. C 18, 3497 (1985).

${ }^{15}$ J. W. Edwards and P. E. Parris, Phys. Rev. B 40, 8045 (1989).

${ }^{16}$ P. E. Parris, J. Stat. Phys. 65, 1161 (1991). 